\title{
Author Index Vol. 3,1996
}

Adorno.D. 213 Aguilera,G. 311(A) Andreoni, G. 364 Anzai,J. 254 Araki,S. 336 Arzt,E. 28,227 Asian, T. 313(A) Assies, J. 318(A) Auer,K.von 318(A)

Bany.U. 247

Bauer, J. 317(A)

Bekker,M. 318(A)

Benigni, F. 364

Berghella, A.M. 213

Berman.A.S. 141

Black, P.H. 141

Bokut,T. 316(A)

Bonsall.R. 47,56

Burakowski, T. 247

Buske-Kirschbaum, A. 314 (A), 318 (A)

Cardinali, D J*. 102 Carlson, N.G. 289 Casciani, C.U. 213 Castrill6n,P. 102 Chancellor-Freeland, C. 141 Chautard,T. 62 Chiappelli, F. 371 Chover-Gonzalez, A.J. 358 Cizza,G. 93

Clow, A. 318 (A), 319 (A) Conde,G.L. 358 Conn,C.A. 239 Conti,A. 325 Corsten,M. 318(A) Costas,M. 227 Cutrera,R. 102

Danilova,T. 316(A) Dayan,C.M. 316(A) Deb, S. 69 Deinzer.R. 312(A) DelBeato,T. 213 Demissie, S. 233 DePotter,W. 119 DeSouza,E.B. 205 Dijkstra, CD. 311(A) DiLoreto, S. 213 Di Marco, F. 213 Dinan,T.G. 319(A) 
Di Santo, E. 285 Downing, J.E.G. 317(A)

Eisel,U. 317(A) Emery, M. 47,56 Esquifino, A.I. 102 Evans, P.D. 318(A) Exton,M.S. 320(A)

Feleder.C 76,342 Fincher, E.F., IV 82 Foddi, M.C. 285 Fratelli,M. 364

Gabellec,M.-M. 304 GabreiI,H. 318(A) Gahring,L.C. 289 Gaillard, R.C. 62 GalH,G. 364 Ganea, D. 35 Geiben,A. 318 (A), 320(A) Ghanta,V.K. 233 Ghezzi,P. 285,364 Giovambattista, A. 62 Goldbach,J.M. 315(A) Goodkin,K. 318(A) Gottschall, P.E. 69 Grif\&amp;is,R. 304 Grossmann,A. 310 GruzelierJ. $312(\mathrm{~A})$

Habu, S. 254

Hadid,R. 62

Haour,F. 304

Harbuz,M.S. 310,315 (A), 316(A),

Hart,R.P. 35

Hashimoto, K. 205

Heier,P. 317(A)

Hellhammer, D.H. 314(A), 318(A),

320 (A) Henderson, D. 312(A) Higuchi,K. 319(A) Hiramoto, N.S. 233 Hiramoto, R.N. 233 Hirst, W.D. 311 (A) Hoadley,M.E. 313(A) Hodgson, D.M. 371 Holsboer,F. 227 Hopkins, S.J. 313(A) Hori,T. 135 iiBen, S.B. 320 (A) Hosoi,M. 135 
Hucklebridge, F. 318 (A), 319 (A)

Huitinga,I. 311(A)

IannottijF. 312(A) Ingram, CD. 316(A) Inoue,S. 219 Irwin, M. 310(A)

Jafarian-Tehrani, M. 304

Janiak,R. 247

Jarry,H. 76,342

Jessop,D.S. 315(A), 316 (A), 358

Jobst,S. 314(A)

Jones, S.B. 119

Kalyanaraman, V.S. 56 Kawanishi, K. 87 Kimura,M. 219 Kirschbaum, C 314 (A), 320 (A) Kiss, A. 311(A) Klemeidam,C 312(A) Kluger,M.J. 239 Koillas,G. 317(A) Kozak,W. 239 Krueger,J.M. 82

Kulmer.R.A. 289 Kunert-Radek, J. 131 Kung,M. 371

$m b M .56$

Lambert, S.E. 319(A)

Laskowska-Bozek, H. 247

Lassmann, H. 317 (A)

Leon,L.R. 239

Leonhardt, S. 76

Lewartowski, B. 247

Lightman, S.L. 314(A), 315 (A), 316(A),

358 Lindholm,D. 317(A) Luheshi.G. 313(A)

McCann,S.M. 321 McCormack, R.J. 35 Maestroni, G.J.M. 325 Makino, S. 205 Marchetti,B. 1 Marti, O. 315(A) Mathews, H.L. 119 Mennini,T. 285 Mepham,J.A. 312(A) Minto.M. 364 Misiewicz,B. 93

KARGER. (C) 1997 S.Karger AG, Basel

E-Mail karger@karger.ch Fax+4161 3061234 
Moguilevsky,J.A. 76, ^P" Moore, P.M. 314(A) Morrow, N.S. 371 Murzenok,P. 316(A)

Nagano, I. 205

Nagel,E. 320(A) Nagy,A. 312(A) Nahmod,V.E. 227 Nakata,A. 336 Neumann, O. 320(A) N igawara, T. 254 Niimi,M. 87

Oka,K. 135 Oka,T. 135

Pabst,M.J. 82 Paez Pereda, M. 227 Parrott,R.F. 313(A) Pawlikowski, M. 131 Pellegrini, P. 213

Perez Castro, C. 227 Perks, P. 314 (A), 316 (A) Pfizemnaier, K. 317(A) Pinxteren,J. 119 Pirke, K.-M. 320 (A) Pringle,A.K. 312(A)

Quan,N. 47,56

Rauh,W. 314(A) Rayboume, R.B. 93 Refojo, D. 342 Renshaw,D. 358 Rezneick,R.H. 319(A)

Ricciaxdi-Castagnoli, P. 285 Richards, P.J. 319(A) Roberts, J.C. 119 Rogers, S.W. 289

Rojavin,M.A. 333 Roth, J. 313 (A), 315 (A) RothwelU.C. 319(A) 
Rothwell,N. 310(A) Rudolph, K. 239 Ryzewski,J. 247

Sacco, S. 364 Sakurai.S. 336 Salmona, S. 364 Sato,M. 87

Schedlowski, M. 320(A) Schmidt, E.D. 311(A) Schoneberg, A. 317 (A) Sch(iller,N. 312(A)

Schult,M. 320(A) Schutz,S. 318(A) Shanks, N. 314(A), 316(A) Smith, C.C. 323 Smith, F.

312(A) Smith, T. 310 Snelson, C.L. 316(A) Solito,E. 311(A) Soszynski,D. 239 Spinedi,E. 62

Stalla,G.K. 28,227 Stepieri, H. 131 Steinmuller, C. 320(A) Sternberg, E.M. 93,323 Storr,B.

313(A) Streilein,J.W. 112 Stypula,G. 131 Sundstrom, L.E. 312(A)

Takahara,J. 87 Takao,T. 205 Takebe, K. 254 Takemura, T. 205 Tanigawa, T. 336 Tatro,J.B. 259

Taylor, A.N. 371 Taylor, A. W. 112,195 ThakoreJ.H. 319(A) Tilders, F.J.H. 311(A) Tio,D.L.

371 Tojo,C. 205 
Tripp, A. 313(A) Tsygankov, A.Y. 333 Tsykhun,G. 316(A)

Van Dam, A.M. 311(A) Vellucci, S.V. 313(A) Vezzani,A. 364

Vikentjeva, N. 316(A) Vingerhoets, A.J.J.M. 318(A) Virag,L. 317(A) Vbge,J. 320(A)

VonAuer,K. 314(A)

Wada,Y. 87 Wakugawa,Y. 135 Watanobe.H. 254 Watkins,A. 310 Weidenfeld.J. 352 Weis,S.

314(A) Weiss, J.M. 47,56 Westermann, J. 320(A) Wilde, G.J.C. 312(A) Wilkin, G.P. 311(A)

Windle,R.J. 316(A) Wolf,0. 320(A) Wurst,W. 317(A) Wustmans, A. 314(A) Wuttke, W. 76, 342

Yirmiya,R. 352 Yokoyama, M. 336 Young, K.A. 311(A)

Zeisberger, E. 313 (A), 315 (A) Zelazowska, E. 93 Zhang, S.-Q. 219 Zhang, Z. 47, 56 Zhu,G. 141

Zhytkevich, T. 316(A) Zinetti,M. 364 Ziskin,M.C. 333 Zylinska,K. 131

Author Index 
Neuroimmunomodulation Vol. 3,1996 